\title{
ULTRASONIC EVALUATION OF INTRATHORACIC MASSES
}

\author{
by Alan H. Wolson, MD
}

\begin{abstract}
$B$ mode ultrasound can be used to evaluate intrathoracic masses that contact the chest wall. It provides a noninvasive technique to distinguish cystic from solid lesions and to assess the internal structure. Four cases are reported in which this procedure was used to provide diagnostic information.
\end{abstract}

Indexing Words

B Scanning
Ultrasound

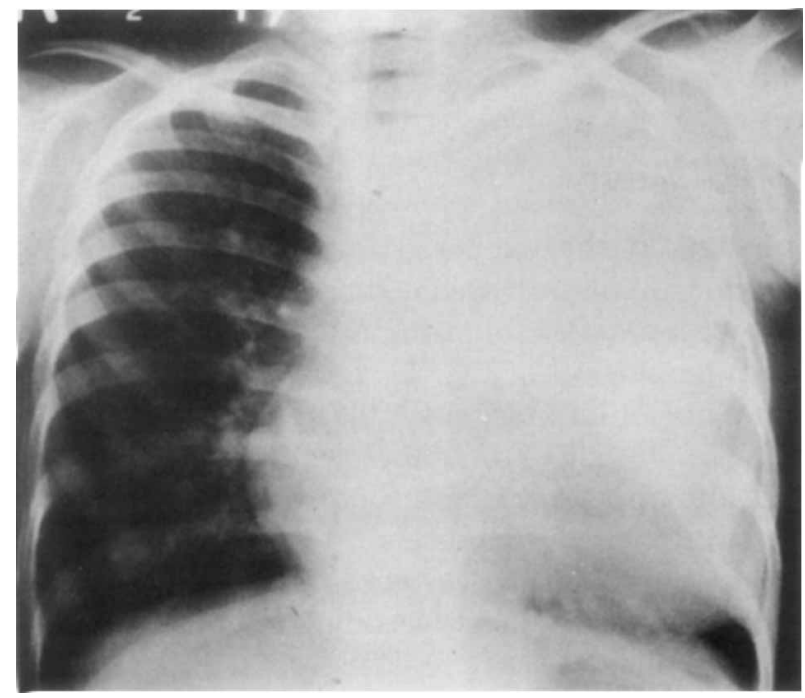

FIGURE 1. PA chest radiograph demonstrates a large smoothly marginated mass occupying most of the left hemithorax. No mediastinal shift has occurred, and there is some normally aerated lung posteriorly.

Chest radiographs (Fig. 1) were interpreted as showing opacification of the left upper lobe and lingula with compression of the left lower lobe which had partially reexpanded in comparison with previous studies. There was no mediastinal shift and this was interpreted as a left upper lobe mass.

An ultrasound examination of the left hemithorax (Fig. 2) revealed a large sonolucent lesion with multiple septae. The lesion remained sonolucent at high gain and there was excellent through transmission. No motion was detected over this lesion but typical cardiac motion was 


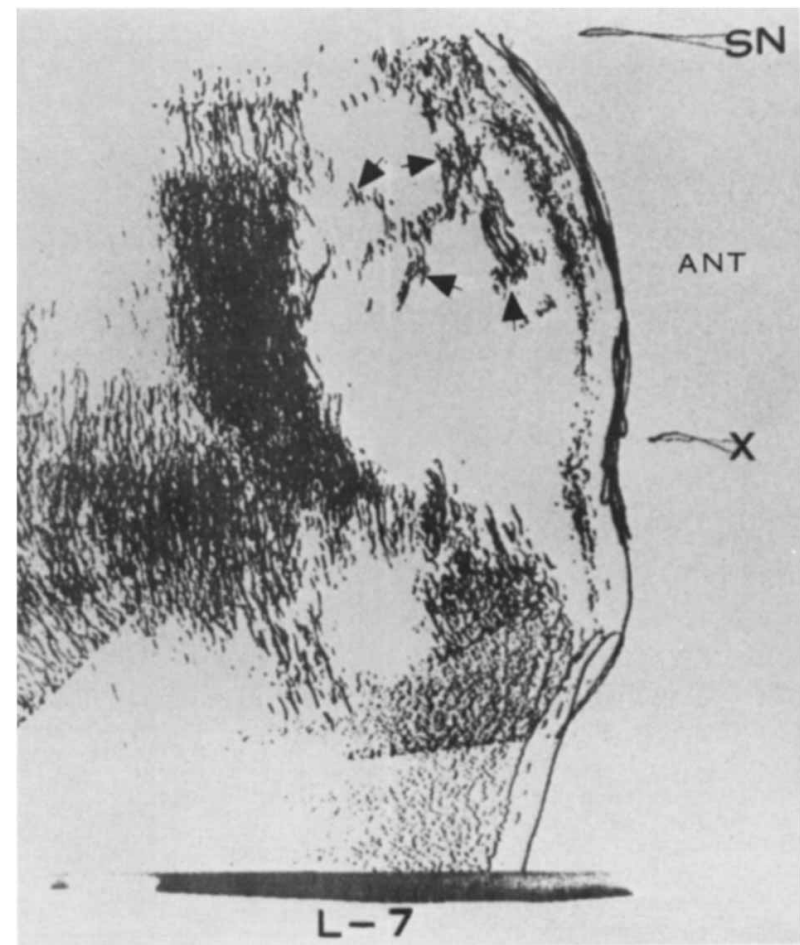

FIGURE 2. A longitudinal (sagittal) section $7 \mathrm{~cm}$ to the left of midline reveals a large sonolucent mass, with good through transmission and multiple septae (arrows). ( $S N=$ suprasternal notch, $X=$ Xiphoid)

detected in the expected location. The appearance of this lesion was considered to be consistent with a cystic hygroma or cystic teratoma.

An angiogram (Fig. 3) revealed neither systemic nor pulmonary arterial supply to the mass. Only small caliber branches went to a rudimentary left upper lobe, and there was marked compression of the LLL vessels.

Based on the ultrasound and angiographic examinations, the mass was diagnosed as an avascular, multiloculated, cystic lesion extrinsic to the left lung.

The mass was completely resected and the left lung was reexpanded. The pathological examination revealed a thin-walled, multiloculated cystic hygroma.

Patient 2, a 46 year old white female in good health, was found to have a diaphragmatic mass on routine chest radiographs. Comparison with previous examination done six years earlier revealed that the mass had approximately doubled in size. The mass was located anterolaterally on the left hemidiaphragm; a diagnostic pneumoperitoneum (Fig. 4) demonstrated that the lesion was above the diaphragm. The differential diagnostic considerations were cystic lesions versus tumor.

An ultrasound examination was performed with the patient in the supine and right lateral

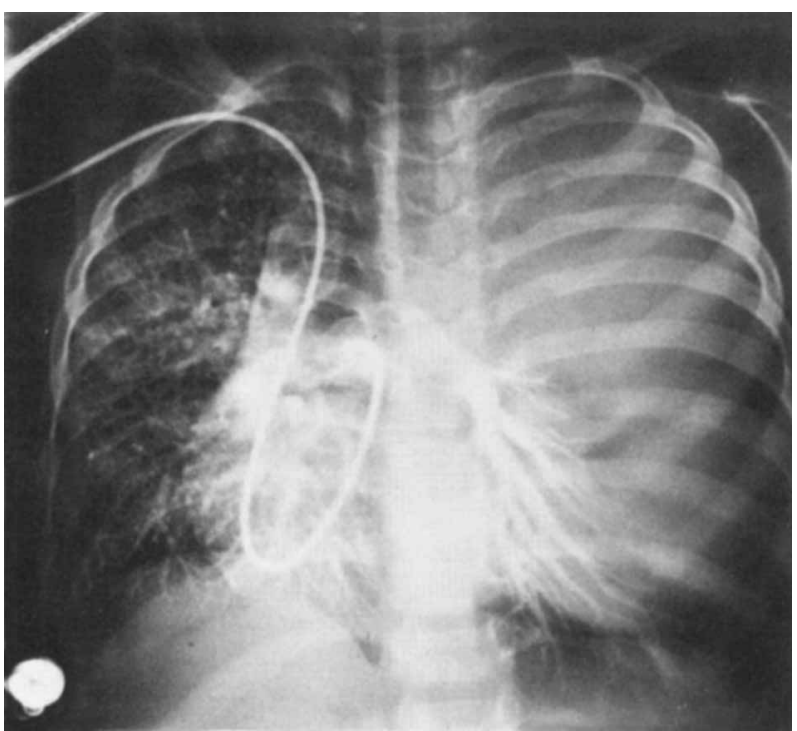

FIGURE 3. Pulmonary arteriogram showing displacement of pulmonary vessels by the large mass and absence of vascular supply to the mass.

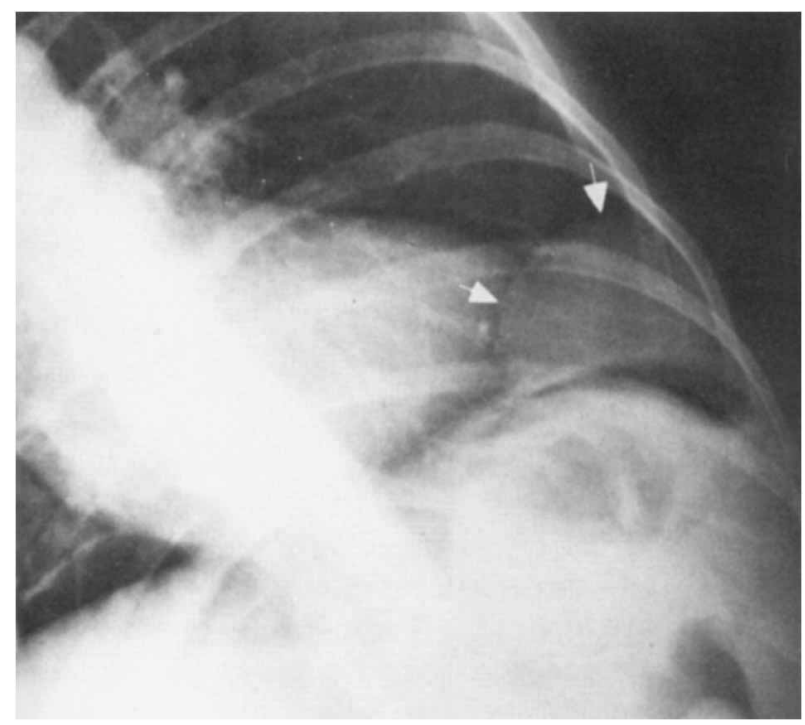

FIGURE 4. An oblique view from the diagnostic pneumoperitoneum demonstrates the lesion (arrows) above the diaphragm and shows the anterolateral location.

decubitus positions (Fig. 5A and B). These examinations revealed a sonolucent lesion with good through transmission. This cystic appearance was considered most suggestive of a pleural or pericardial cyst.

A thoracotomy was performed and a coelomic cyst, thought to be pericardial, was removed.

Patient 3 is a 27 year old white female who was first seen by a physician in July 1975 , because of an upper respiratory infection accompanied by a productive cough. A chest radiograph revealed a right anterior mediastinal mass in the right cardiophrenic angle. This was not investigated further. 


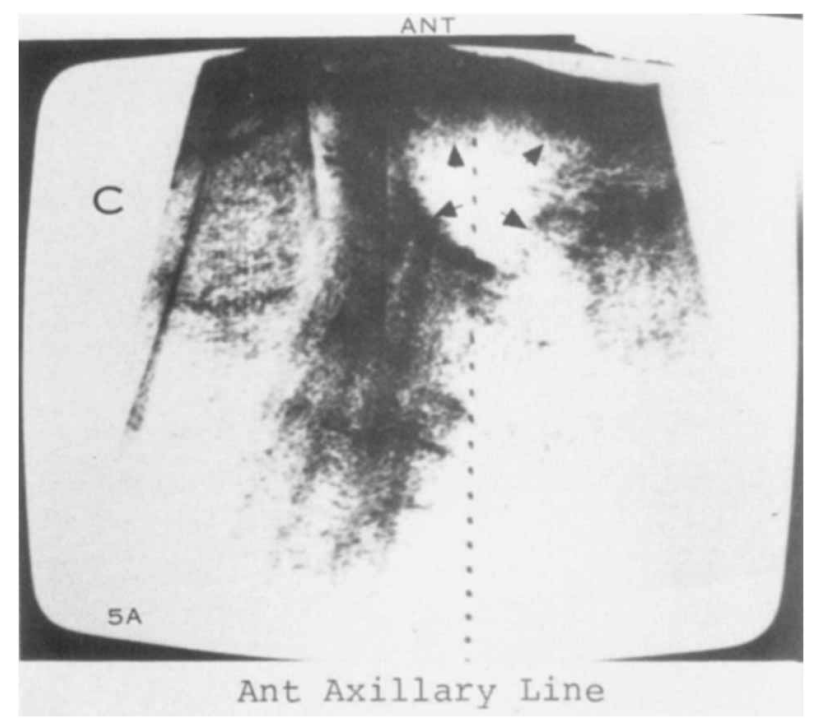

5A

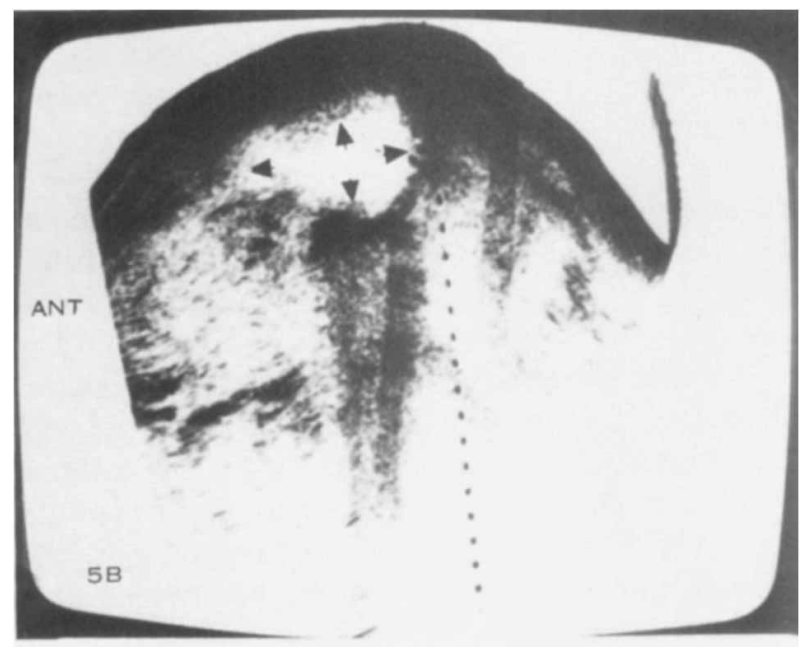

Rt Lat Decub

5B

FIGURE 5, A and B. Supine (5A) and right lateral decubitus (5B) views reveal the mass (arrows) to be sonolucent with good through transmission and strong backwall echoes. $(\mathrm{C}=$ cephalad)

In October 1975 , a preemployment chest radiograph again revealed the mass, and the patient was evaluated at another hospital. The lesion did not move with cardiac pulsation or diaphragmatic motion. No other abnormalities were detected.

The patient was then referred to UMMC where a chest radiograph (Fig. 6) was unchanged from previous studies. A B mode ultrasound examination revealed a septated sonolucent lesion (Fig. 7) adjacent to the right side of the heart. No motion of the cyst walls or septum was observed on either A mode or $M$ mode.

Surgery revealed a multiloculated pericardial cyst.

Patient 4, a 66 year old white female, was referred to UMMC in May 1974 for treatment of a

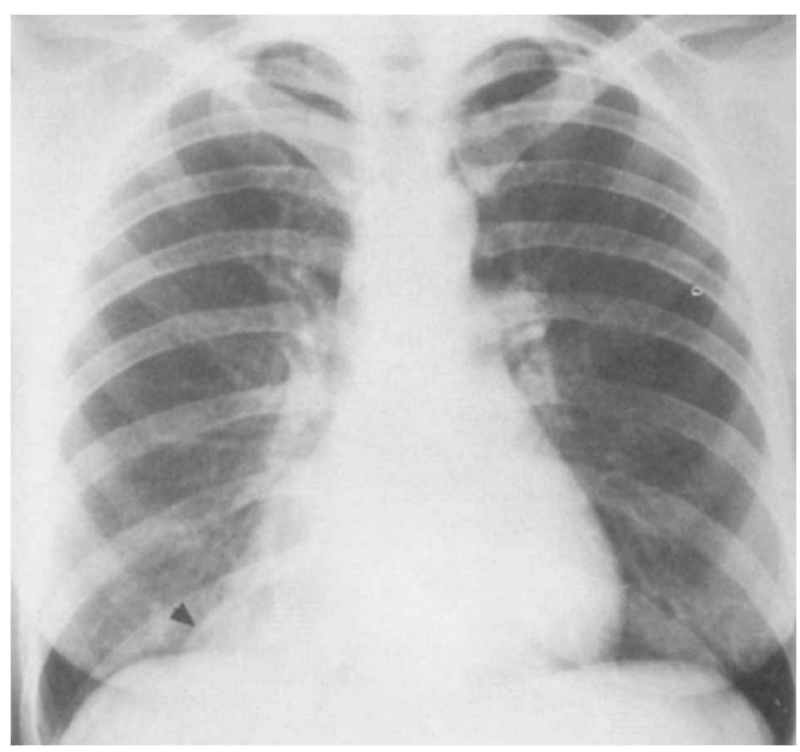

FIGURE 6. PA chest radiograph shows a small soft tissue density (arrow) in the right cardiophrenic angle.

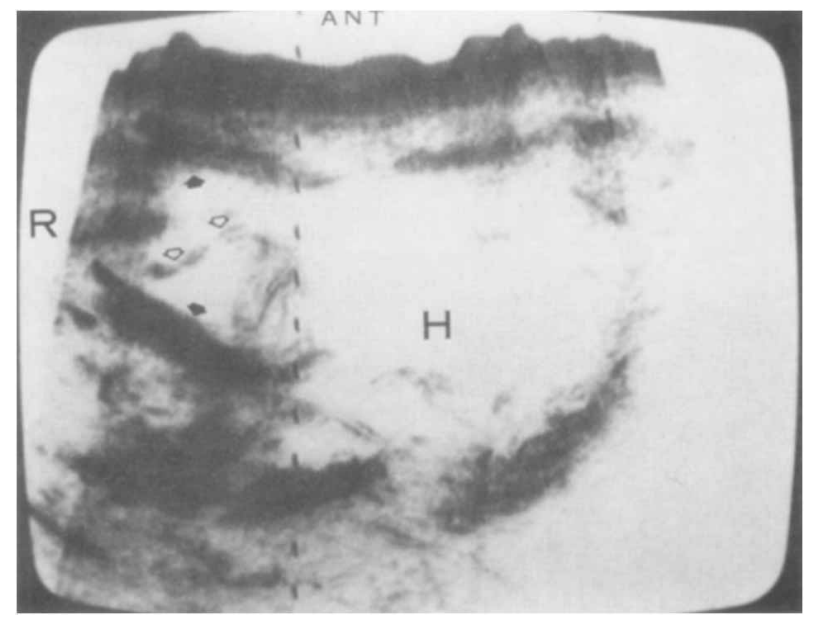

FIGURE 7. A B scan of the chest $3 \mathrm{~cm}$ above the xiphoid shows a septated sonolucent lesion (closed arrows) adjacent to the heart. $(H=$ heart; open arrows - septum)

presumed pericardial cyst. Six years earlier a mass had been discovered in the right cardiophrenic angle and was watched by her physician. Two weeks prior to admission to the UMMC, she was hospitalized elsewhere because of hypotension. At that time the mass was noted to have enlarged. She complained of dyspnea on exertion and chest pain.

A chest radiograph (Fig. 8) at UMMC revealed a mass in the right cardiophrenic angle consistent with a pericardial cyst. An ECG gated (R-wave triggered) B scan of the heart (Fig. 9) was performed and demonstrated a sonolucent lesion adjacent to the heart. On right heart catherterization, the mass did not opacify. Clear fluid was aspirated from the mass (cytology 


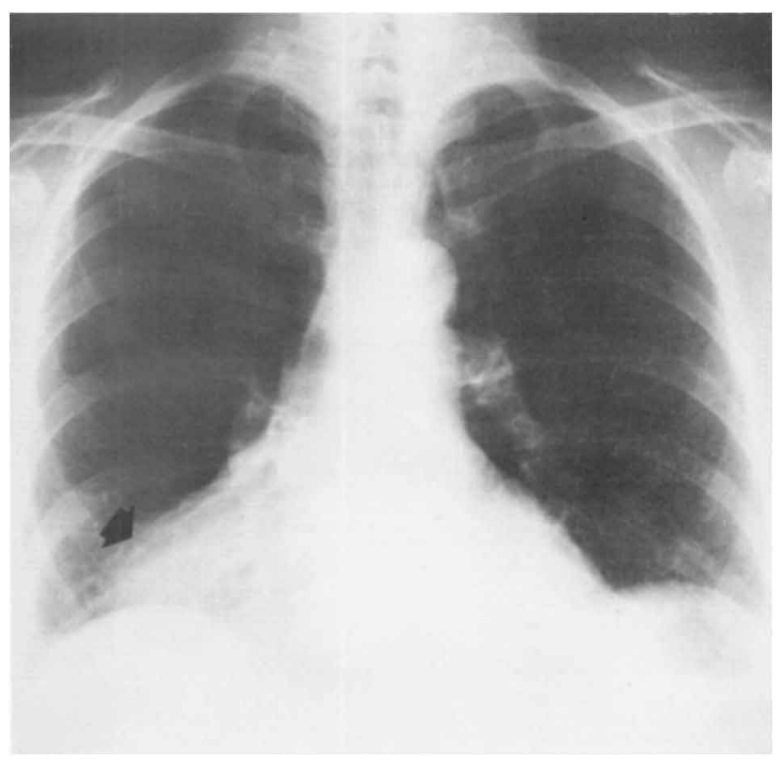

FIGURE 8. PA chest radiograph shows a large soft tissue density (arrow) in the right cardiophrenic angle.

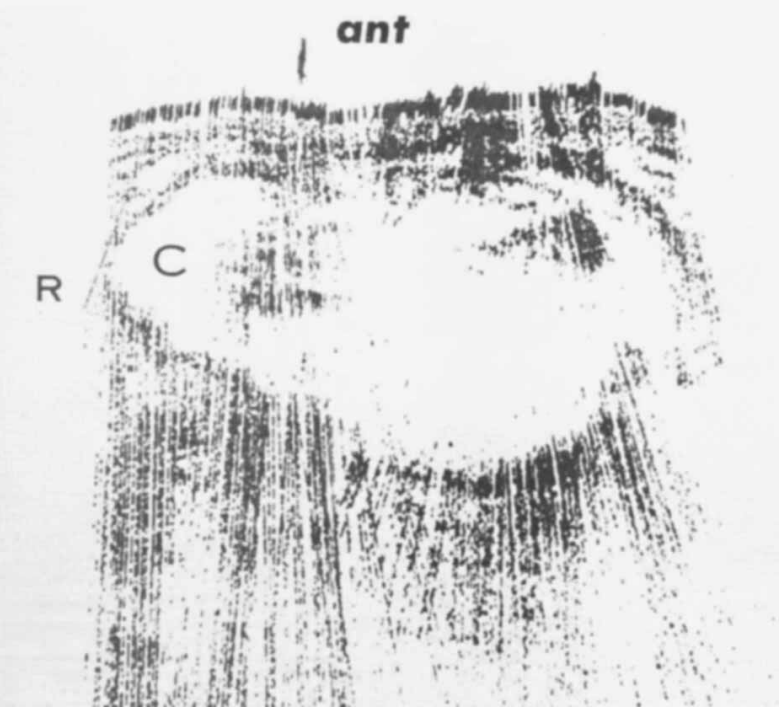

FIGURE 9. An R-wave triggered $B$ scan of the heart deınonstrated a sonolucent (esion adjacent to the heart. $(\mathrm{C}=\mathrm{cyst})$

negative) and contrast medium was injected (Fig. 10) to outline the lumen.

The consensus was that this was a benign pericardial cyst, and no surgery was performed because of the patient's other medical problems.

\section{DISCUSSION}

Diagnostic ultrasound has had limited application for evaluation of diseases of the chest, with the notable exception of the heart. Only two reports have discussed the application of $\mathrm{B}$ mode ultrasound to the chest and both dealt with evaluation of pleural opacities $(1,4)$. Prior reports discussed the use of $\mathrm{A}$ and/or $\mathrm{M}$ mode

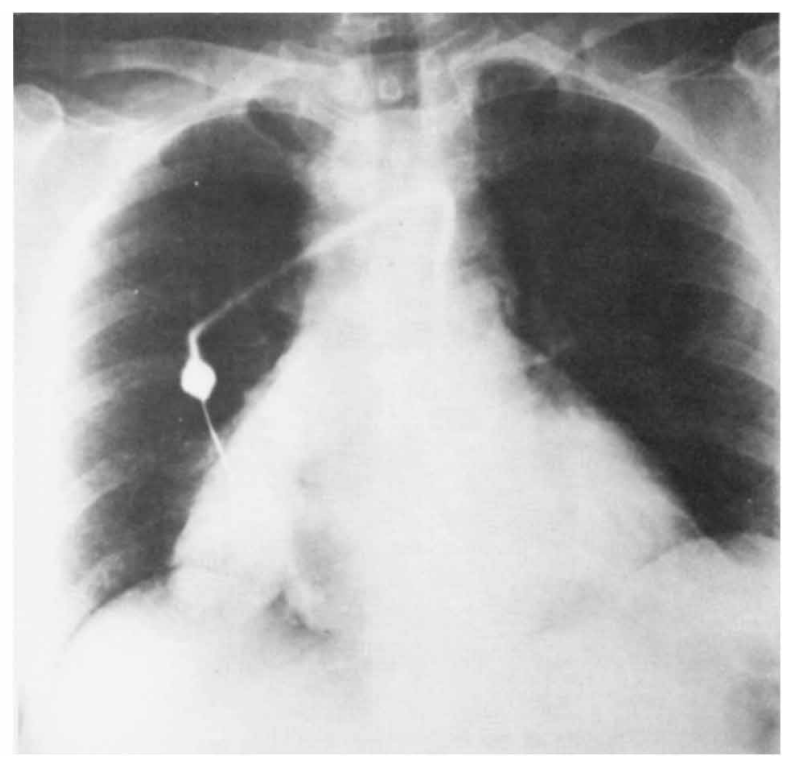

FIGURE 10. Contrast medium injected percutaneously into the cyst outlines the lumen.

ultrasound for evaluation of pleural disease (2, 3 ), tumors, mediastinal masses (6), fluid-filled cavities (7), pneumonia (8), and pulmonary emboli (9).

B mode ultrasound can also be used to examine masses that are in contact with the chest wall to differentiate between solid and cystic lesions.

In the patients reported above, ultrasound provided the first definite evidence that the lesions were cystic. In Patient 1, considerable confusing information was available from the child's previous hospitalization and from his admission at UMMC. The ultrasound study revealed that the entire mass seen on the chest radiograph was cystic. In addition, the presence of multiple septa strongly suggested a diagnosis of cystic hygroma or cystic teratoma.

Patient 2 presented a diagnostic dilemma since the lesion was known to be a slowly growing mass, and the list of differential diagnoses for diaphragmatic masses is quite long (10). Metastatic disease was not considered since the lesion had been present six years and the patient was completely asymptomatic. However, a slowly growing benign or malignant primary tumor was considered, as was a cystic lesion. The ultrasound study clearly identified a unilocular cyst.

The radiographs in Patients 3 and 4 were more straightforward. In both a sharply defined mass was seen in the right cardiophrenic angle and the most likely etiology was a pericardial cyst. However, solid masses may occur here, including prominent epicardial fat pad, teratoma, 
and thymolipoma. The ultrasound examination in both cases confirmed that the lesion was cystic and in one case demonstrated the multilocular nature of the lesion.

\section{SUMMARY}

The applications of B scanning to the thorax remain limited because of the problem of reflection by the lung surface. However, when a lesion seen on a chest radiograph is in contact with the chest wall, ultrasound can be effectively used, not only to determine whether the lesion is cystic or solid, but also to outline its size and define its anatomic characteristics.

\section{REFERENCES}

1. Doust B, Baum JK, Maklad NF, and Doust VL: Ultrasonic evaluation of pleural opacities. Radiology 114: $135,1975$.

2. Gryminski J: Use of ultrasound method in diagnosis of pleural diseases. Gruzlica 39: 694, 1971.
3. Joyner CR Jr, Herman RJ, and Reid JM: Reflected ultrasound in the detection and localization of pleural effusion. JAMA 200: 399, 1967.

4. Sandweiss DA, Hanson JC, Gosink BB, and Moser $\mathrm{KM}$ : Ultrasound in diagnosis, localization, and treatment of loculated pleural empyema. Ann Intern Med 82: 50, 1975.

5. Goldberg BB: Suprastemal ultrasonography. JAMA 215: 245, 1971.

6. Goldberg BB: Mediastinal ultrasonography. J Clin Ultrasound 1: 114, 1973.

7. Naas W, Schentke KU, and Renger F: Moglichkeiten der eindimensionalen Ultraschall diagnostik von Tumocen und flussigkeitgeffulten Hohlraumen in Abdomen and Thorax. 2 Aerztl Fortbild (Jena) 67: $72,1973$.

8. Bogin IuN, Mutina ES, Bodganov AV, et al: Ultrasound diagnosis of pneumonia. Klin Med (Mosk) 48: $123,1970$.

9. Joyner CR Jr, Miller LD, Dudrick SJ, et al: Reflected ultrasound in the detection of pulmonary embolism. Trans Assoc Am Phys 79: 262, 1966.

10. Anderson LS and Forrest JV: Tumors of the diaphragm. Am J Roentgenol 119: 259, 1973. 